\title{
World Glaucoma Week in Port Harcourt: Comparison of Yield and Public Awareness of Glaucoma Services in two Consecutive Years
}

\author{
AA Onua and EA Awoyesuku* \\ Department of Ophthalmology, University of Port Harcourt, Nigeria \\ *Corresponding Author: EA Awoyesuku, Department of Ophthalmology, University \\ of Port Harcourt, Nigeria.
}

DOI: 10.31080/ASOP.2020.03.0104
Received: February 12, 2020

Published: February 29, 2020

(C) All rights are reserved by AA Onua and EA

Awoyesuku .

\begin{abstract}
Background: Glaucoma is a leading cause of irreversible blindness worldwide. Ignorance and poverty are known factors for late presentation. Early diagnosis and treatment are indispensable tools in curbing the menace of visual impairment from glaucoma. The Rivers State branch of the Ophthalmological Society of Nigeria (OSN) creates awareness and undertakes screening of the populace for glaucoma during World Glaucoma Weeks observed annually. The aim of this study is to compare the socio-demographics of participants, the yield of glaucoma patients during the case finding exercises and the most effective public awareness campaign method. Materials and Method: Cross-sectional survey of 173 clients presenting for glaucoma screening in 2018 and 2019 World Glaucoma Weeks at the ophthalmology clinic of the University of Port Harcourt Teaching Hospital. Socio-demographic characteristics, pattern of referral, medium of awareness, educational status and occupation of the study-participants were obtained and analyzed.

Results: One hundred and three participants were females. The mean age of the study participants was $39.3 \pm 14.6$ years. Age group ( 40 - 49 years) had the highest proportion of participants (25.4\%). The yield was $7.5 \%$. Forty participants in 2018 were informed of the glaucoma screening exercise by first-degree relatives. In the 2019 edition, $73(42.2 \%)$ of the clients were referred through radio while 50 (28.9\%) were referred through posters.

Conclusion: The yield of glaucoma cases was 7.5\%. Our study shows that dissemination of information related to glaucoma to members of the public could be better via first degree relatives, radio announcements and posters displayed at strategic locations.
\end{abstract}

Keywords: Awareness; Screening for Glaucoma; World Glaucoma Week

\section{Introduction}

Late presentation of glaucoma constitutes a significant factor for the visual impairment and blindness often seen among glaucoma patients in Sub-Sahara Africa. Lack of glaucoma awareness, positive family history and illiteracy were associated with late presentation of glaucoma [1]. The awareness and knowledge of existing glaucoma services helps in providing access to quality care, more understanding of the disease, leading to better control and prevention of early onset of blindness from glaucoma. Screening of the general population for glaucoma is a veritable tool in identify- ing potential glaucoma patients for further confirmatory investigations and commencement of early expert management. Adekoya., et al. in a study in South-West Nigeria observed the need to intensify present efforts aimed at increasing public awareness, emphasizing the irreversible nature of the disease, as well as encouraging at-risk groups such as first-degree relatives to go for screening [1].

Mass public awareness campaign and screening had been observed to impact positively on early detection of glaucoma [1,2]. Furthermore, the positive impact of frequent free eye camps or- 
World Glaucoma Week in Port Harcourt: Comparison of Yield and Public Awareness of Glaucoma Services in two Consecutive Years

ganized by different non-governmental organizations, especially in rural areas, cannot be over-emphasized. Glaucoma suspects are usually referred to our eye clinic from such eye camps. Apart from improved awareness, the level of education of patients and patients' first-degree relatives have been noted to have positive relationship with earlier detection of glaucoma [2,3]. Events undertaken by ophthalmologists and other eye care practitioners during World Glaucoma Weeks at various community levels also increase availability of ophthalmology services, counseling and screening and referral of ocular morbidities to specialist centers [3]. The importance of these activities during the World Glaucoma Weeks in rural and suburban areas cannot be over-emphasized. More awareness campaigns are necessary in order to educate glaucoma patients and communities, and counseling services should be made available to families of sufferers. Educating the general public on the need for early presentation to ophthalmologists and not to other health practitioners may make early diagnosis and management feasible, hence improving the prognosis of the disease.

\section{Materials and Methods}

This was a cross-sectional survey of clients presenting for glaucoma screening during the 2018 and 2019 editions of World Glaucoma Week at the Ophthalmology clinic of the University of Port Harcourt Teaching Hospital (UPTH). Socio-demographic characteristics, pattern of referral, medium of awareness, educational status and occupation of the study-participants were obtained. In 2018, glaucoma patients undertaking treatment in UPTH were requested to invite their first-degree relatives for glaucoma screening. In the 2019 edition, invitation of the public was given out via radio announcement and jingles, social media and posters displaced in different strategic points in Port Harcourt municipality. Assessment of the Vertical Cup Disc Ratio of the Optic nerve head was used to determine glaucomatous disc (VCDR of 0.8 - 1.0); Glaucoma Suspect (VCDR > 0.5 - 0.7) for glaucoma was done by the use of indirect ophthalmoscope. All data were cross checked for accuracy, entered into a proforma and were analyzed using commercially available statistical data management software- Statistical Package for Social Sciences (IBM-SPSS) version 25. Distribution was described as mean and standard deviation. Continuous variables were reported with tables. Analysis of Variance (ANOVA) was used to determine the statistical significance of the differences between proportions. The level of significance was taken to be $\mathrm{p}<0.05$.

\section{Consent and ethical clearance}

Ethical clearance was obtained from the Ethical Committee of
University of Port Harcourt Teaching Hospital. Informed written consent and assent were obtained from each client before enrolment into the study in accordance with Helsinki Declaration involving human subjects.

\section{Results}

A total of 173 clients participated in the study, 103 (59.5\%) were females. Male to female ratio was 1:1.5. The mean age of the study participants was $39.3 \pm 14.6$ years; with age range of 6 - 83 years. Age group ( 40 - 49 years) had the highest proportion of participants (25.4\%). The differences in the proportion of age groups between the male and female genders were not statistically significant $(\mathrm{p}=0.794)$.

\begin{tabular}{|l|c|c|c|}
\hline Age Group (Years) & Male (\%) & Female (\%) & Total (\%) \\
\hline$<20$ & $5(2.9)$ & $8(4.6)$ & $13(7.5)$ \\
\hline $20-29$ & $12(6.9)$ & $15(8.7)$ & $27(15.6)$ \\
\hline $30-39$ & $14(8.1)$ & $28(16.2)$ & $42(24.3)$ \\
\hline $40-49$ & $17(9.8)$ & $27(15.6)$ & $44(25.4)$ \\
\hline $50-59$ & $12(6.9)$ & $16(9.3)$ & $28(16.2)$ \\
\hline 60 and Above & $10(5.8)$ & $9(5.2)$ & $19(11.0)$ \\
\hline Total & $70(40.5)$ & $103(59.5)$ & $173(100)$ \\
\hline \multicolumn{4}{|l|}{ Pearson's Chi- Square $=2.381 \mathrm{df}=5 \mathrm{p}=0.794$} \\
\hline
\end{tabular}

Table 1: Age and Gender Distribution of the Study Participants in the Two Years.

In 2018 edition of World Glaucoma week, a total of 40 participants were screened for glaucoma. The Mean age of participants was $36.3 \pm 14.7$ years, with age range of 10 - 68years. Male to female ratio was 1:1.4. Participants of 30-39 years constituted the highest (30\%).

\begin{tabular}{|l|c|c|c|}
\hline Age Group (Years) & Male (\%) & Female (\%) & Total (\%) \\
\hline $0-19$ & $2(5.0)$ & $4(10.0)$ & $6(15.0)$ \\
\hline $20-29$ & $3(7.5)$ & $4(10.0)$ & $7(17.5)$ \\
\hline $30-39$ & $5(12.5)$ & $7(17.5)$ & $12(30.0)$ \\
\hline $40-49$ & $2(5.0)$ & $5(12.5)$ & $7(17.5)$ \\
\hline $50-59$ & $3(7.5)$ & $1(2.5)$ & $4(10.0)$ \\
\hline 60 and Above & $2(5.0)$ & $2(5.0)$ & $7(17.5)$ \\
\hline Total & $17(42.5)$ & $23(57.5)$ & $40(100)$ \\
\hline
\end{tabular}

Table 1a: Age and Gender Distribution of the Study Participants in 2018. 
World Glaucoma Week in Port Harcourt: Comparison of Yield and Public Awareness of Glaucoma Services in two Consecutive Years

In 2019 edition of World Glaucoma week, a total of 133 participants were screened for glaucoma. The Mean age of participants was $42.3 \pm 14.6$ years with age range of 6-83. Male to female ratio was 1:1.6. Participants of 40 - 49 years constituted the highest (25.5\%) (Table 1b).

\begin{tabular}{|l|c|c|c|}
\hline Age Group (Years) & Male (\%) & Female (\%) & Total (\%) \\
\hline $0-19$ & $3(2.3)$ & $4(3.0)$ & $7(5.3)$ \\
\hline $20-29$ & $9(6.8)$ & $11(8.2)$ & $20(15.0)$ \\
\hline $30-39$ & $9(6.8)$ & $21(15.8)$ & $30(22.6)$ \\
\hline $40-49$ & $12(9.0)$ & $22(16.5)$ & $34(25.5)$ \\
\hline $50-59$ & $9(6.8)$ & $15(11.3)$ & $24(18.1)$ \\
\hline 60 and Above & $10(7.6)$ & $8(6.0)$ & $18(13.5)$ \\
\hline Total & $52(39.2)$ & $81(60.8)$ & $133(100)$ \\
\hline
\end{tabular}

Table 1b: Age and Gender Distribution of the Study Participants in 2019.

Table 2 shows that the 40 participants in 2018 were informed of the glaucoma screening exercise by their family relatives who were glaucoma patients receiving various glaucoma services offered in the Ophthalmology clinic of University of Port Harcourt Teaching Hospital. In the 2019 edition of the program, 73 (42.2\%) of the clients were referred through radio while 50 (28.9\%) were referred through poster. Client referral from various social media was $9(5.2 \%)$ while only $2(1.2 \%)$ clients participated as a result of information from family members who are glaucoma patients.

\begin{tabular}{|c|c|c|c|c|c|}
\hline Year & $\begin{array}{c}\text { Patient } \\
\text { (\%) }\end{array}$ & $\begin{array}{c}\text { Poster } \\
(\%)\end{array}$ & $\begin{array}{c}\text { Radio } \\
\mathbf{( \% )}\end{array}$ & $\begin{array}{c}\text { Social } \\
\text { media } \\
\text { (\%) }\end{array}$ & Total (\%) \\
\hline 2018 & $40(23.1)$ & - & - & - & $40(23.1)$ \\
\hline 2019 & $2(1.2)$ & $50(28.9)$ & $7342.2)$ & $9(5.2)$ & $133(76.9)$ \\
\hline Total & $42(24.3)$ & $50(28.9)$ & $73(42.2)$ & $9(5.2)$ & $173(100)$ \\
\hline
\end{tabular}

Table 2: Comparison of Pattern of referral of clients in the Study Population.

Table 3 shows that 103 (59.5\%) of the study participants were civil /public servants, 36 (20.8\%) were students, 25 (14.5\%) were business men and women while retirees constituted $4 \%$ and applicants were $1.6 \%$ of the participants.

Table 4 shows that 107 (61.8\%) study participants had tertiary education, 51 (29.5\%) had secondary education while 15 (8.7\%) of the clients had primary education. The difference in the educa- tional status of the participants in the various groups was not statistically significant ( $\mathrm{p}=0.192)$.

\begin{tabular}{|l|c|c|c|c|c|}
\hline Occupation & $\mathbf{2 0 1 8}$ & $\mathbf{( \% )}$ & $\mathbf{2 0 1 9}$ & \% & Total (\%) \\
\hline Business & 5 & $(2.9)$ & 20 & $(11.6)$ & $25(14.5)$ \\
\hline Civil service & 18 & $(10.4)$ & 85 & $(49.1)$ & $103(59.5)$ \\
\hline Retiree & 4 & $(2.3)$ & 3 & $(1.7)$ & $7(4.0)$ \\
\hline Student & 13 & $(7.5)$ & 23 & $(13.3)$ & $36(20.8)$ \\
\hline Applicants & 0 & $(0)$ & 2 & $(1.6)$ & $2(1.6)$ \\
\hline Total & 40 & $(23.1)$ & 133 & $(76.9)$ & $173(100)$ \\
\hline
\end{tabular}

Table 3: Comparison of Study participants by Occupation.

\begin{tabular}{|l|l|l|l|l|l|}
\hline Education & $\mathbf{2 0 1 8}$ & $\begin{array}{l}\text { Per- } \\
\text { cent- } \\
\text { age }\end{array}$ & $\mathbf{2 0 1 9}$ & $\begin{array}{l}\text { Per- } \\
\text { cent- } \\
\text { age }\end{array}$ & Total (\%) \\
\hline Tertiary & 21 & $(12.1)$ & 86 & $(49.7)$ & $107(61.8)$ \\
\hline Secondary & 13 & $(7.5)$ & 38 & $(21.9)$ & $51(29.5)$ \\
\hline Primary & 6 & $(3.5)$ & 9 & $(5.2)$ & $15(8.7)$ \\
\hline $\begin{array}{l}\text { Total Pear- } \\
\text { son's Chi } \\
\text { Square= } \\
\text { 3.300 }\end{array}$ & 40 & $(23.1)$ & $\begin{array}{l}133 \\
\mathrm{P}=0.192\end{array}$ & $(76.9)$ & $173(100)$ \\
\hline
\end{tabular}

Table 4: Comparison of Study Participants by Educational Level.

Table 5 shows that a total of 13 clients (7.5\%) were screened positive for glaucoma, out of which number, 9 were harvested in 2018. The difference in the VCDR among the various participants in this study was statistically significant.

\section{Discussion}

The World Glaucoma Association subcommittee on screening advocates screening for glaucoma as a means of creating public awareness of the disease and creating an avenue for detection of vision disorders in medically underserved communities [4].

Glaucoma is a leading cause of blindness and is generally asymptomatic until late in the disease, therefore early diagnosis an essential part of the control strategy [5].

Gender

More females accessed the free screening programme from our study even though this was not statistically significant when compared. 
Females have been known to access free health programmes more probably because of economic disadvantages they are unable to pay for fee-paying health programmes [6,7]. There is no clear gender predilection for open angle glaucoma; but women tend to outlive men and represent $60 \%$ of all glaucoma cases combined, there is also some evidence that the female sex hormone might be protective of the optic nerve [7].

Age

Participants of 40 - 49 years constituted the highest (25.5\%) number of participants in our study when comparing the two years.

Age is an established risk factor for glaucoma [8]. In a study by Song., et al. [9] comparing two cohorts of patients referred for glaucoma screening the mean age of participants were 55.7 and 51.7years respectively. Al-Aswad., et al. [10] while screening high risk population had the highest age range of 40 - 64years and more female than male participants. In a community-based screening for glaucoma in Nigeria the highest age range of participants presenting were 41- 50years [11] while in South western Nigeria the mean age of participants for screening was 61.9years [9] and 56.3years [12].

\section{Referral route/ occupation of participants}

In 2018 our participants were referred mainly through other patients while in 2019, other modes of referral were used radio constituting the highest (42.2\%). In both years the highest numbers of participants were civil servants $59.5 \%$. In glaucoma screening program in South Eastern Nigeria radio was the commonest mode of referral and business /self-employed people consisted of the highest number of participants [13] In a study on the role of mass media in the creation of awareness for glaucoma, it was found that mass media had a low impact on glaucoma awareness except during Glaucoma awareness week [14]. The recommendation is for this to be on a continuous basis.

\section{Glaucoma yield}

A total of 13 clients (7.5\%) were screened positive for glaucoma, out of which number, 9 were harvested in 2018. The difference in the VCDR among the various participants in this study was statistically significant. This study showed a low yield of glaucoma from the entire population but it is noteworthy that most of the confirmed glaucoma cases were from the cohort of first-degree family relatives. This agrees with the consensus statement by the World Glaucoma association that glaucoma screening is more vi- able if targeted at glaucoma patient relatives [15]. This was also buttressed by a study by Nathaniel G. [16] who had a prevalence of glaucoma of $13.7 \%$ for offspring of glaucoma patients and $5.3 \%$ for siblings of glaucoma patient during an opportunistic glaucoma screening for first degree relations.

\section{Conclusion}

Glaucoma screening is a very important means of early detection of glaucoma patients and finding out the population that at risk. Mass public campaigns using first degree relatives, radio announcements and posters displayed at strategic locations could be effective in our environment.

\section{Bibliography}

1. Adekoya BJ., et al. "Glaucoma in southwest Nigeria: clinical presentation, family history and perceptions". International Ophthalmology 34 (2014): 1027-1036.

2. Enock ME., et al. "Glaucoma in a Suburban Tertiary Care Hospital in Nigeria". Journal of Ophthalmic and Vision Research 5.2 (2010): 87-91.

3. Omoti AE. "Glaucoma in Benin-city, Nigeria”. Nigerian Postgraduate Medical Journal 12 (2005): 189-192.

4. World Glaucoma association. Screening for open angle glaucoma.

5. Kreft D., et al. "Prevalence, incidence, and risk factors of primary open-angle glaucoma- a cohort study based on longitudinaldata from a German public health insurance". BMC Public Health 19 (2019): 51.

6. Merzel C. "Gender differences in health care access indicators in an urban, low-income economy". American Journal of Public Health 90.6 (2000): 909-916.

7. Vajaranant TS., et al. "Joslin CE". Current Opinion in Ophthalmology 21.2 (2010): 91-99.

8. Guedes G., et al. "Glaucoma and aging". Current Aging Science 4.2 (2011): 110-117.

9. Song YJ., et al. "Comparison of glaucoma patients referred by glaucoma screening versus referral from primary eye clinic". PLoS ONE 14.1 (2019): e0210582.

10. Al-Aswad LA., et al. "Screening for glaucoma in populations at high risk: The eye screening of New York project". Journal Cogent Medicine (2017): 4. 
11. Awoyesuku EA and Chukwuka IO. "Is community based case detection of glaucoma relevant? Intraocular Pressure level and vertical cup disc ratioof participants at a screening programme in a rural setting in Nigeria". Journal of Clinical and Experimental Ophthalmology 2 (2018): 022-026.

12. Olushola O., et al. Spectrum of Glaucoma presentation in Suburban Teaching Hospital in South Western Nigeria.

13. Olawoye 0., et al. "Evaluation of community eye outreach programs for early glaucoma detection in Nigeria". Clinical Ophthalmology 7 (2013): 1753.

14. Kizor-Akariwe NN., et al. "Awareness and Knowledge about glaucoma and proportion of people with glaucoma in an urban outreach programme in Southeast Nigeria". BMJ Open Ophthalmology 1.1 (2017): e000018.

15. Rewri P., et al. "Role of MassCommunication and Health Care in promoting Glaucoma awareness: An Observational study". Delhi Journal of Ophthalmology 18 (2018): 36-39.

16. Nathaniel GI and Awoyesuku EA. "Opportunistic screening for Glaucoma amongst Glaucoma patients first degree relatives who accompanied them to Glaucoma clinic". International Annals of Medicine 1.4 (2017): 3.

\section{Assets from publication with us}

- Prompt Acknowledgement after receiving the article

- Thorough Double blinded peer review

- Rapid Publication

- Issue of Publication Certificate

- High visibility of your Published work

Website: www.actascientific.com/

Submit Article: www.actascientific.com/submission.php

Email us: editor@actascientific.com

Contact us: +919182824667

Citation: AA Onua and EA Awoyesuku. "World Glaucoma Week in Port Harcourt: Comparison of Yield and Public Awareness of Glaucoma Services in two Consecutive Years". Acta Scientific Ophthalmology 3.3 (2020): 52-56. 